\title{
Erratum
}

\section{A Differential Geometric Criterion for Moishezon Spaces}

\author{
Robert Frankel \\ Department of Mathematics, University of Massachusetts, Boston, MA 02125, USA
}

Math. Ann. 241, 107 (1979)

\section{Moishezon Spaces}

The purpose of this note is to correct an error in reference [1]. All of our notation and definitions will be consistent with that reference.

We recall the statement of Proposition $1 b$ :

Proposition $1 \mathrm{~b}$. Let $\mathscr{L}$ be a pseudonegative sheaf over a compact analytic space $X$. Let $(S, U, h)$ be pseudonegative data for $\mathscr{L}$. If $\operatorname{codim} S \geqq 2$, then $H^{1}(X, \mathscr{L})=0$ and $H^{1}\left(X, \mathscr{L}^{*} \otimes K(X)\right)=0$.

Takeo Ohsawa has pointed out that our proof of this result is incorrect, because the harmonic projection operator is not support-preserving. At present, we have only the following result with which to replace Proposition $1 \mathrm{~b}$ :

Proposition. Let $X$ be a compact analytic space. There exists a pseudonegative sheaf $\mathscr{L}$ over $X$ such that $H^{1}(X, \mathscr{L})=0$.

We will see in the proof that $\mathscr{L}$ can always be taken to be a sheaf of the type constructed in Proposition 3.

Proof. Let $\pi: Y \rightarrow X$ be a projective desingularization of $X$, and let $E$ be the exceptional set in $Y$. If $M$ is any negative line bundle over $Y$, then by Proposition 3, we know that $\pi_{*}(M \otimes \mathcal{O}(-E))$ is a pseudonegative sheaf on $X$. But let's assume that, in fact, $M$ is so negative that $H^{1}(Y, M \otimes \mathcal{O}(-E))=0$. [For example, this will be true if $M \otimes \mathcal{O}(-E)$ is negative, by Kodaira's Vanishing Theorem.]

Let $\mathscr{L}=\pi_{*}(M \otimes \mathcal{O}(-E))$. We will show that $H^{1}(X, \mathscr{L})=0$. Let $\left\{U_{i}\right\}$ be a locally finite open cover of $X$, and let $\left\{\alpha_{i j}\right\}$ be a 1-cocycle with respect to this cover. We want to show that there exists a 0 -cocycle $\left\{\beta_{i}\right\}$ with respect to our open cover such that $\alpha_{i j}=\beta_{j}-\beta_{i}$. But $\left\{\pi^{-1} U_{i}\right\}$ is a locally finite open cover of $Y$, and by definition of $\pi_{*}$, the cocycle $\left\{\alpha_{i j}\right\}$ gives rise in canonical fashion to a cocycle (which we denote $\left.\left\{\alpha_{i j}^{\prime}\right\}\right)$ on $\left\{\pi^{-1} U_{i}\right\}$. Furthermore, since (as is well-known) the natural map from $H^{1}\left(\left\{\pi^{-1} U_{i}\right\}, M \otimes \mathcal{O}(-E)\right)$ to $H^{1}(Y, M \otimes \mathcal{O}(-E))$ is one-to-one, there exists a 
cocycle $\left\{\beta_{i}^{\prime}\right\}$ such that $\alpha_{i j}^{\prime}=\beta_{j}^{\prime}-\beta_{i}^{\prime}$. But now we use the definition of $\pi_{*}$ once more: $\left\{\beta_{i}^{\prime}\right\}$ gives rise canonically to $\left\{\beta_{i}\right\}$, a 0 -cocycle relative to $\left\{U_{i}\right\}$ which satisfies $\alpha_{i j}=\beta_{j}-\beta_{i}$.

\section{References}

1. Frankel, R.: A differential geometric criterion for Moishezon spaces. Math. Ann. 241, 107-112 (1979)

Received June 7, 1980 